\title{
On Experimental Determination of Gradient Coefficient
}

\author{
B.R. Murphy', I.E. Eleftheriadis ${ }^{2}$, J. Ning, W. Milligan ${ }^{3}$ and E.C. Aifantis ${ }^{2,3}$ \\ 'Information Products Inc., 414 East Fortieth Street, Holland, MI 49423 USA \\ ${ }^{2}$ Aristotle University of Thessaloniki, Polytechnic School, 54124 Thessaloniki, Greece \\ ${ }^{3}$ Center for Mechanics of Materials and Instabilities, Michigan Technological University, \\ Houghton, MI 49931 USA
}

\begin{abstract}
A simple, direct method to measure the value of the gradient coefficient is discussed. Since the gradient term in gradient theories of plasticity and elasticity accounts for the heterogeneity of deformation, it is possible to estimate the non-standard gradient coefficient through a simple inhomogeneous deformation field. Pure bending tests of rectangular beams made of high purity aluminum (>99.5\%) with a grain size gradient were thus designed and conducted. By measuring the curvature of the deformed beam, the gradient coefficient can be directly determined.
\end{abstract}

\section{INTRODUCTION}

One of the basic problems in using the gradient theory of plasticity developed by Aifantis and coworkers $/ 1-7 /$ is the determination of the gradient coefficient in its yield condition. In $/ 8,9 /$, values of this coefficient for different materials were estimated theoretically based on nonlocal concepts and the self-consistent method, which pointed out that the grain size of the material has a strong effect. An indirect experimental method $/ 10$ / was also developed by matching numerical analysis of gradient approach with the measurement of the width of stationary shear bands. However, it can be difficult to measure accurately the width of shear bands and this method may be not generally applicable.

\section{EXPERIMENTAL PROCEDURES}

A plate of high-purity aluminum was machined in a step-wise fashion on a horizontal milling machine (Figure 1). Residual stresses introduced during machining were eliminated and the grain structure was 
stabilized by a low temperature annealing (573K for 8 hours). Cold-rolling was performed in multiple passes until a final plate thickness of $11.5 \mathrm{~mm}(0.451 \mathrm{in})$ was achieved. Bend bars were cut from the cold-rolled plate and polished to the following nominal dimensions: length $\mathrm{L}=140 \mathrm{~mm}(5.5 \mathrm{in})$, width $\mathrm{w}=12.5 \mathrm{~mm}(0.5$ in), and height $2 \mathrm{~h}=11 \mathrm{~mm}(.430 \mathrm{in})$. All bend bars used in the experiment were placed in the same oven batch at $873 \mathrm{~K}$ for 2 hours. The resulting recrystallized grain structure is shown in Figure 2 . The grain size in the heavily cold-worked $(22.2 \%)$ section of the bend bars is $1 \mathrm{~mm}$, while that of the $7.8 \%$ cold-worked section is $10 \mathrm{~mm}$. Grain sizes are approximated from the micrograph shown in Figure 2.

Pure bending experiments were performed in a standard four-point-bend fixture equipped with 6 linear voltage displacement transducers (LVDTs) (Figure 3). The samples were carefully positioned in the fixture,

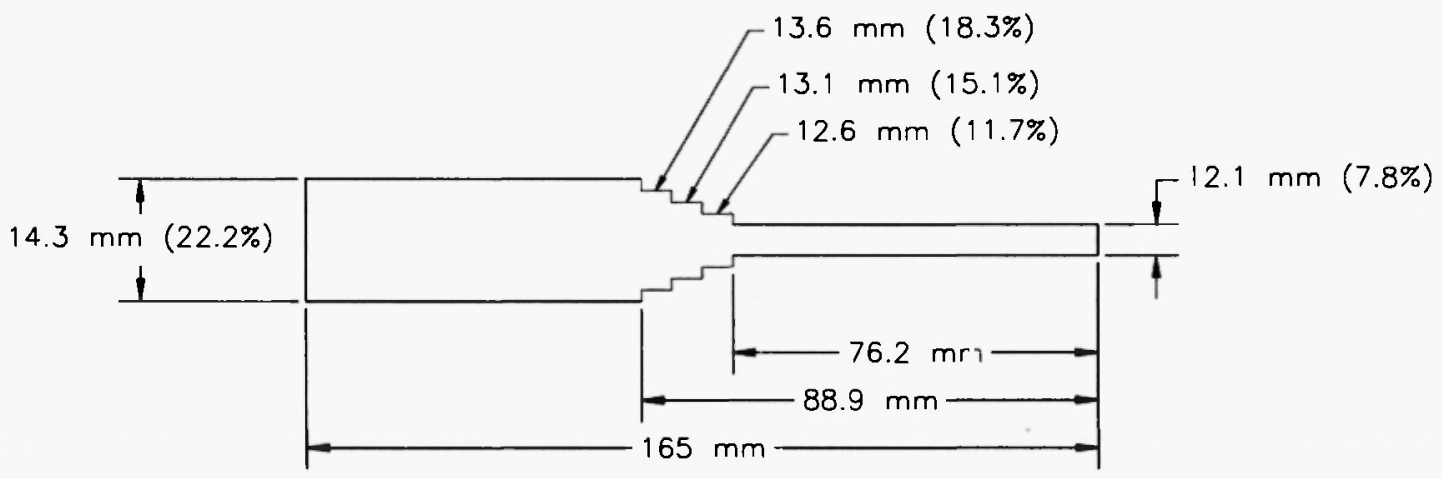

Fig. 1: Dimensions of the high purity aluminum plate prior to cold-rolling and the subsequent cold-rolling percentages for each section

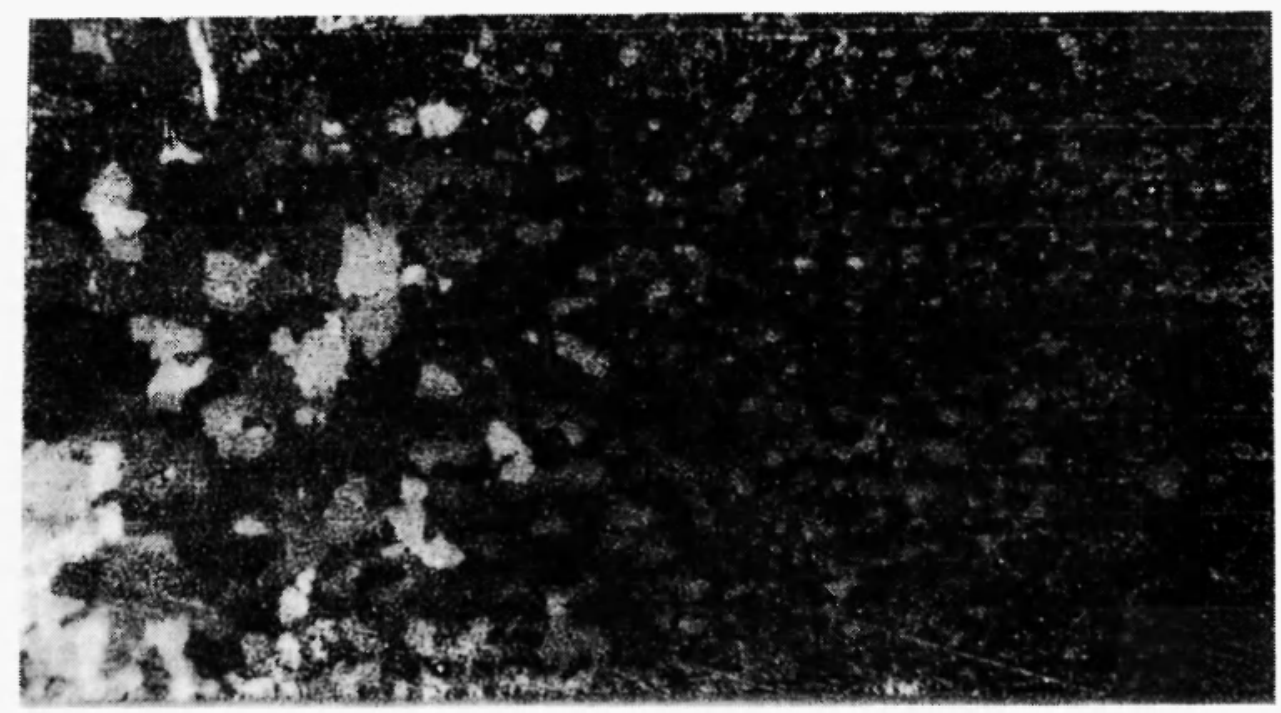

Fig. 2: Optical micrograph of the gradient area illustrating the variation of grain size along the sample axis 
with the center of the gradient zone located over LVDT 4 . The center of the sample was located midway between LVDT 3 and 4 . The samples were incrementally loaded in $\sim 2.25 \mathrm{~kg}(5 \mathrm{lb})$ increments and LVDT voltage readings were recorded. Using preconstructed calibration curves for each LVDT, displacement values were directly determined from the LVDT voltage data. Load was recorded by a computer connected to a standard load cell. From the load readings the corresponding applied moments $\mathrm{M}$ can be calculated.

Figure 4 presents the deflection data for a sample over each LVDT at various loads. Knowing that the beam center is between LVDT 3 and 4, we would expect the maximum deflection to be between LVDT 3 and 4 for a homogeneous beam. Due to the inhomogeneous nature of the beams manufactured for these experiments, the maximum deflection is shifted towards LVDT 3.

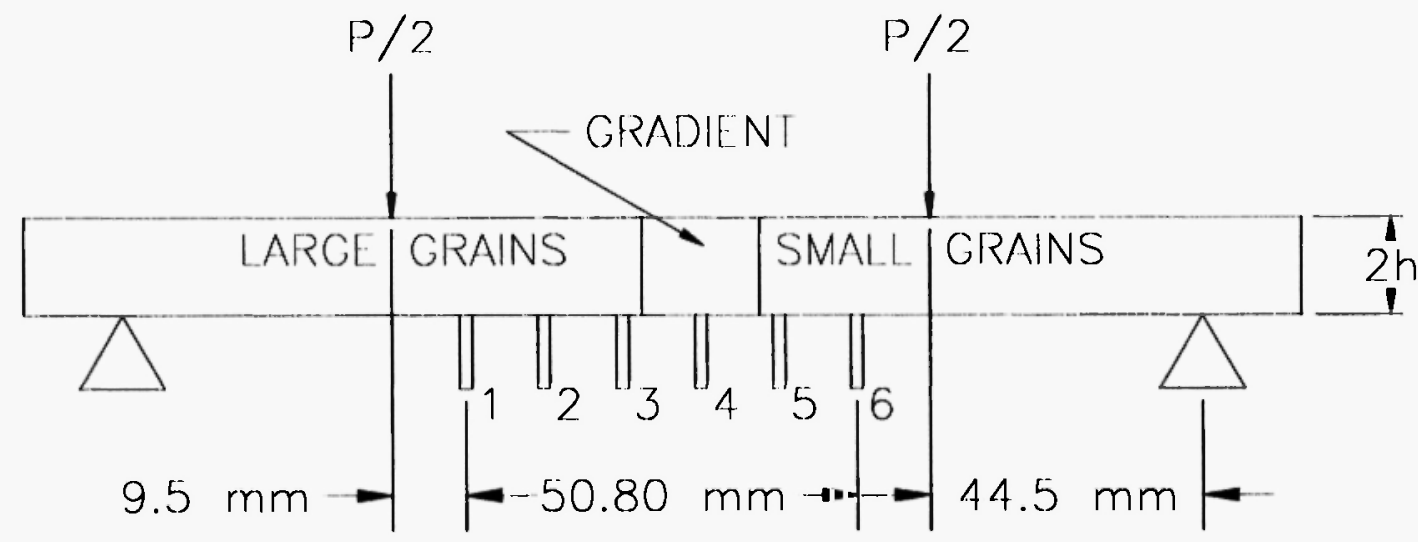

Fig. 3: Illustration of the 4-point bending test configuration

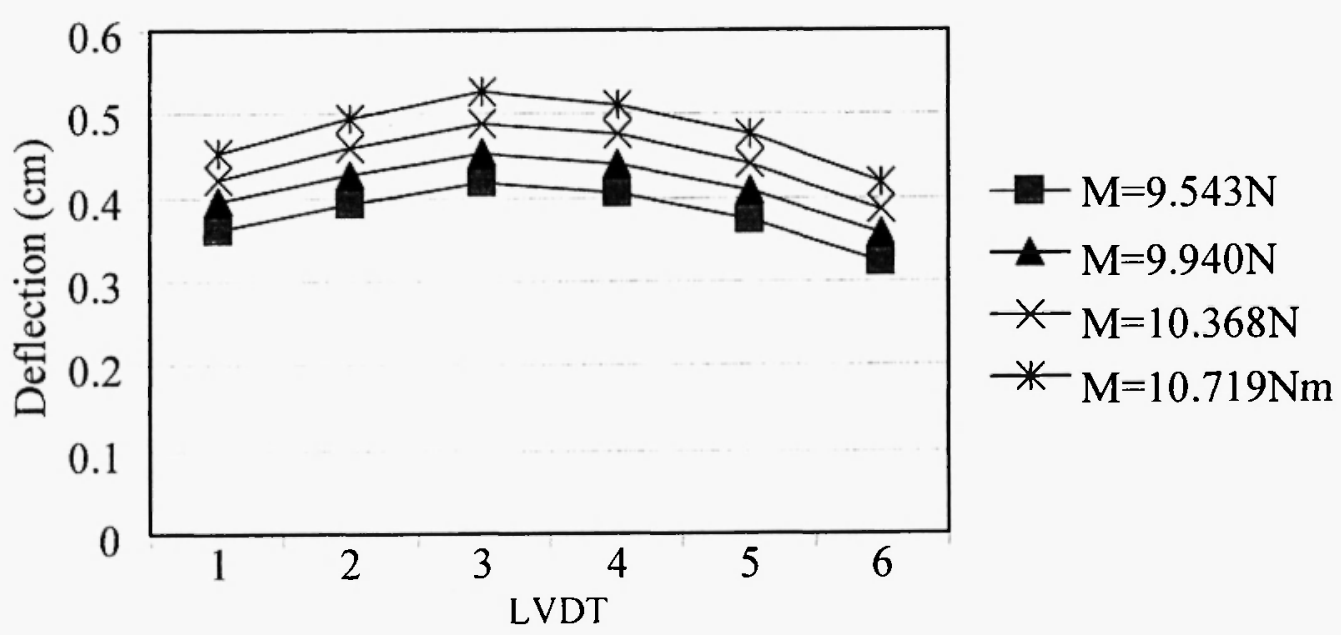

Fig. 4: Asymmetric profile of deflection data 


\section{GRADIENT THEORY OF PLASTICITY}

In order to interpret the heterogeneity of plastic deformation including shear band widths and spacing, the gradient theory of plasticity was proposed by Aifantis and coworkers /1-7/ based on the following modification of the yield condition

$$
\tau=k(\gamma)+c \nabla^{2} \gamma
$$

where $k(\gamma)$ is the flow stress representing the isotropic hardening effects of the homogeneous deformation. $\tau$ denotes the equivalent von Mises shear stress $\tau=\sqrt{\sigma_{i j}^{\prime} \sigma_{i j}^{\prime} / 2}, \gamma$ is the equivalent shear strain $\gamma=\sqrt{2 \varepsilon_{i j}^{\prime} \varepsilon_{i j}^{\prime}}$ and $c$ is the gradient coefficient. The deviatoric stress and strain are respectively denoted by $\sigma_{i j}^{\prime}$ and $\varepsilon_{i j}^{\prime}$. Equation (1) has been used to estimate the width of shear bands by properly choosing the value of the gradient coefficient $c / 5 /$.

It is also noted that self consistent arguments may be employed to correlate $c$ with the grain size, the elastic material parameters and the hardening/softening parameters in the expression of $k(\gamma)$. The latter depend upon the deformation history, environment, and fabrication of the material. On the other hand, the gradient coefficient could be experimentally determined via an inhomogeneous deformation field where the local stress, strain and the strain gradient can be easily measured or calculated. According to these requirements, pure bending tests with an inhomogeneous deformation field were designed as already described in Section 2.

\section{PURE BENDING DEFORMATION OF A BEAM}

The deformation in a beam under pure bending is one of the simplest inhomogeneous deformation fields. For a beam specimen with a rectangular cross section of width $w$ and height $2 h$, let $x$ denote the coordinate along the axis of the beam with the origin located at the left end of the beam and $y$ is the coordinate vertical to the neutral axis of the cross-section (Figure 5). The deflection of the beam is denoted by $v$. According to the basic assumptions of the beam theory, the axial strain distribution in the cross-section reads

$$
\varepsilon=\kappa y ; \quad \kappa=-v^{\prime \prime}
$$

where $\kappa$ is the curvature of the deformed beam (assumed herein positive when the beam is convex up), while $v^{\prime \prime}$ denotes the second derivative of the deflection $v$ with respect to $x$. For a rigid-plastic material subjected to monotonic loading, the gradient dependent yield condition (1) can be written in uniaxial tension terms as

$$
\sigma=k_{1}(\varepsilon)+3 c \nabla^{2} \varepsilon,(3)
$$




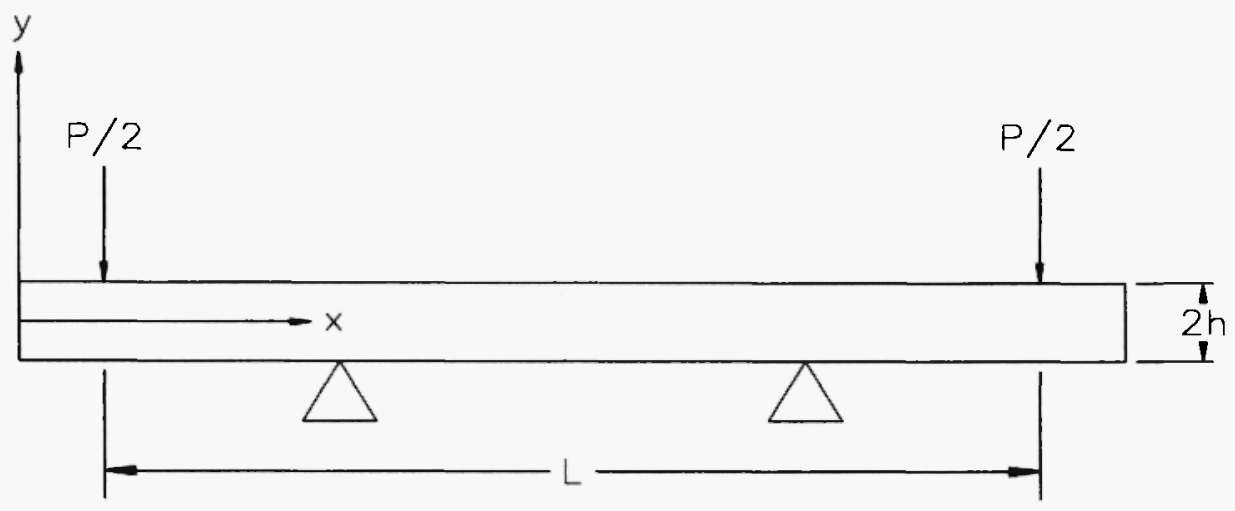

Fig. 5: Coordinate system of the four-point bending test configuration

where $\varepsilon=\gamma / \sqrt{3}$ and $\sigma=\sqrt{3} \tau$ are the equivalent axial strain and stress respectively and $k_{1}(\varepsilon)=\sqrt{3} k(\gamma)$. For a rigidly plastic material, the flow stress $k_{1}(\varepsilon)$ is usually assumed to follow a power law of the form

$$
k_{1}(\varepsilon)=\sigma_{0}\left(\varepsilon+\varepsilon_{0}\right)^{\prime \prime}
$$

with $\sigma_{0}$ and $n$ denoting isotropic hardening parameters. Then, the moment equilibrium condition $(M=$ $\left.\int_{A} \sigma y \mathrm{~d} A\right)$ implies

$$
M=2 w \int_{0}^{h} \sigma y \mathrm{~d} y,
$$

which in view of (3) gives

$$
M=A_{\text {hoon }}+c A_{\text {inh }}\left(\mathrm{d}^{2} \kappa / \mathrm{d} x^{2}\right)
$$

with

$$
A_{\text {lom }}=2 w \int_{0}^{i} k_{1}(\kappa y) y \mathrm{~d} y, \quad A_{\mathrm{inh}}=2 w h^{3}
$$

where $M$ is the bending moment acting on the beam.

From equations (6) and (7), we obtain the following expression for the gradient coefficient

$$
c=\frac{M-A_{\text {loon }}}{A_{\text {inh }}\left(\mathrm{d}^{2} \kappa / \mathrm{d} x^{2}\right)},
$$


provided that the curvature $\kappa$ and its spatial derivative $d \kappa / d x$ vary along the beam. For homogeneous pure bending $\mathrm{K}$ is a constant and then, in view of (2), the gradient term in (3) vanishes. In fact, the determination of $c$ will be accomplished here through the sensitivity of $\kappa$ on the coordinate $x$ as reflected in equation (3).

At the early stages of deformation, the effects of the gradient term are negligible; therefore, the values of the isotropic hardening parameters $\sigma_{0}, n$ and $\varepsilon_{0}$ can be specified at this stage from a uniaxial tension test. The average uniaxial tension test data for the specimen consisting of fine and coarse grain portions, as well as the respective fitting equation, are plotted in Figure 6. The fitting values for the isotropic hardening parameters were found to be $\sigma_{0}=65.42 \mathrm{MPa}, \mathrm{n}=0.21$ and $\varepsilon_{0}=0.0005$. By using the results in Figure 4 , the curvature $\kappa=-\mathrm{d}^{2} v / \mathrm{d} x^{2} \approx-\Delta^{2} v / \Delta x^{2}$ and $\mathrm{d}^{2} \kappa / \mathrm{d} x^{2} \approx-\Delta^{4} v / \Delta x^{4}$ can be calculated in the gradient zone at LVDT 4 using the finite difference method. The results for $\kappa, \mathrm{d}^{2} \kappa / \mathrm{d} x^{2}$, the surface strain $\varepsilon_{s}$ and also the gradient coefficient $c$ calculated by equation (8) are presented in Table 1 . The main advantage of this method is that the gradient coefficient can be calculated near the middle of the beam in the $x$ direction so that possible boundary effects in the differential equation (1) are negligible. The mean value of the gradient coefficient is $c=18 \mathrm{kN}$. An internal length $l=\sqrt{c / G}=0.83 \mathrm{~mm}$ can be calculated, where $G-26 \mathrm{GPa}$ is the shear modulus referred to in the literature for aluminum. It is interesting to notice that this value of $l$ is in the order of magnitude of the grain size.

By considering linear distribution of strain in the $y$ direction, it can be easily proved that $\varepsilon_{e} / \varepsilon_{s}=h_{e} / h$, where $\varepsilon_{p}$ is the maximum elastic strain, $\varepsilon_{s}$ is the surface strain, $h_{e}$ is the half width of the elastic zone and $h$ is the half width of the specimen. From the uniaxial tension data, the maximum elastic strain can be estimated to be approximately $\varepsilon_{c}=0.0005$ and by using the values for $\varepsilon_{s}$ presented in Table 1 , the elastic region height is less than $5.3 \%$ of the specimen height. In the present analysis, only data for large values of the bending moment were taken into account, because the material was assumed to be fully plastic and so the effect of the elastic region near the specimen neutral axis was omitted. In the general case, an elastic-plastic boundary model should be formulated to capture the elastic-plastic mechanical behavior of the material during bending.

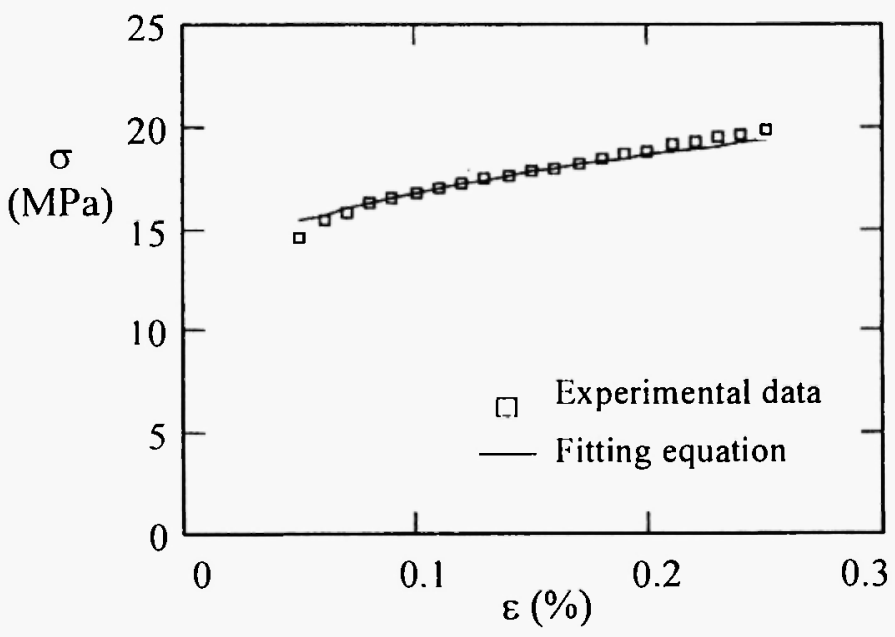

Fig. 6: Tension data at the initial stage of plastic deformation 
Table 1

Curvature $\kappa, d^{2} \kappa / d x^{2}$, surface strain $\varepsilon_{s}$ and gradient coefficient $c$ calculated in the gradient zone at LVDT 4.

\begin{tabular}{|c|c|c|c|c|}
\hline$M(\mathrm{Nm})$ & $\kappa\left(\mathrm{m}^{-1}\right)$ & $\mathrm{d}^{2} \kappa / \mathrm{d} x^{2}\left(\mathrm{~m}^{3}\right)$ & $\varepsilon_{s}=\kappa h$ & $c(\mathrm{kN})$ \\
\hline 9.543 & 1.724 & $2.14910^{4}$ & 0.00948 & 10.81 \\
9.94 & 1.938 & $1.44510^{4}$ & 0.01066 & 19.43 \\
10.368 & 2.112 & $1.60510^{4}$ & 0.01161 & 21.68 \\
10.719 & 2.209 & $2.06510^{4}$ & 0.01215 & 20.03 \\
\hline
\end{tabular}

\section{ACKNOWLEDGMENTS}

The support of US National Science Foundation and EC under the TMR Network project FMRX-CT960062 is gratefully acknowledged.

\section{REFERENCES}

1. E.C. Aifantis. On the microstructural origin of certain inelastic models, ASME J. Engng. Mat. Tech, 106, 326-330 (1984).

2. E.C. Aifantis. The physics of plastic deformation, Int. J. Plasticity, 3, $211-247$ (1987).

3. E.C. Aifantis. On the role of gradients in the localization of deformation and fracture, Int. J. Engng. Sci., 30, 1279-1299 (1992).

4. E.C. Aifantis. Pattern formation in plasticity, Int. J. Engng. Sci., 33, 2161-2178 (1995).

5. H.B. Muhlhaus and E.C. Aifantis. A variational principle for gradient plasticity, Int. J. Solids Struct., 28, 845-858 (1991).

6. I. Vardoulakis and E.C. Aifantis. A gradient flow theory of plasticity for granular materials, Acta Mechanica, 87, 197-217 (1991).

7. H.M. Zbib and E.C. Aifantis. On the gradient-dependent theory of plasticity and shear banding, Acta Mechanica, 92, 209-225 (1992).

8. H.M. Zbib. Strain gradients and size effects in nonhomogeneous plastic deformation, Scripta Metall., 30, 1223-1226 (1994).

9. J. Ning and E.C. Aifantis. Anisotropic and inhomogeneous plastic deformation of polycrystalline solids, in: A.S. Krausz and A.J. Krausz (Eds.), Unified Constitutive Laws of Plastic Deformation, Chapter 7. Academic Press, San Diego, 1996; pp. 319-341.

10. G.L. Panger, Jr., J.L. Ding, H.M. Zbib and E.C. Aifantis. Measurement of shear band characteristics in low carbon steel using photoelasticity, Scripta Metall., 25, 2103-2108 (1991). 
